University of Texas at El Paso

ScholarWorks@UTEP

$12-2014$

\title{
Granularity Helps Explain Seemingly Irrational Features of Human Decision Making
}

Joe Lorkowski

The University of Texas at El Paso, lorkowski@computer.org

Vladik Kreinovich

The University of Texas at El Paso, vladik@utep.edu

Follow this and additional works at: https://scholarworks.utep.edu/cs_techrep

Part of the Computer Engineering Commons

Comments:

Technical Report: UTEP-CS-14-62a

\section{Recommended Citation}

Lorkowski, Joe and Kreinovich, Vladik, "Granularity Helps Explain Seemingly Irrational Features of Human Decision Making" (2014). Departmental Technical Reports (CS). 885.

https://scholarworks.utep.edu/cs_techrep/885

This Article is brought to you for free and open access by the Computer Science at ScholarWorks@UTEP. It has been accepted for inclusion in Departmental Technical Reports (CS) by an authorized administrator of ScholarWorks@UTEP.For more information, please contact Iweber@utep.edu. 


\title{
Granularity Helps Explain Seemingly Irrational Features of Human Decision Making
}

\author{
Joe Lorkowski and Vladik Kreinovich
}

\begin{abstract}
Starting from well-known studies by Kahmenan and Tversky, researchers have found many examples when our decision making seems to be irrational. In this chapter, we show that this seemingly irrational decision making can be explained if we take into account that human abilities to process information are limited; as a result, instead of the exact values of different quantities, we operate with granules that contain these values. On several examples, we show that optimization under such granularity restriction indeed leads to observed human decision making. Thus, granularity helps explain seemingly irrational human decision making.
\end{abstract}

Keywords: Decision making • Granularity $\bullet$ Seemingly irrational behavior

\section{Seemingly Irrational Human Decision Making: Formulation of the Problem}

In the ideal world, people should make perfect decisions. In many real-life situations, we know what is best for us, and we know the exact consequences of each of our actions. In this case, a rational person should select an action that leads to the best possible outcome.

This assumption underlies basic (idealized) economic models: in these models, our decision making may hurt others but every person is interested in selecting a decision which is the best for him/herself.

In the real world, people's decisions are not perfect. In the perfect world, people should make perfect decisions. It is well known, however, that our world is not perfect, and that many people make decisions which are not in their own best interests. People eat unhealthy food, fail to exercise, get drunk, smoke, take drugs, gamble, and do many other things which - as they perfectly know - are bad for their health and bad for their wallets.

Such imperfect decisions can still be described in optimization terms. People engage in all kinds of unhealthy and asocial decision making because they get a lot

J. Lorkowski and V. Kreinovich

Department of Computer Science, University of Texas at El Paso, 500 W. University, El Paso, Texas 79968, USA e-mail: lorkowski@computer.org, e-mail: vladik@utep.edu 
of positive emotions from this engagement. A drug addict may lose his money, his family, his job, his health - but he gets so much pleasure from his drugs that he cannot stop. A gambler may lose all his money, but the pleasure of gambling is so high that he continues gambling (and losing) money until no money is left.

These examples of bad decisions are bad from the viewpoint of a person's health or wealth or social status. In all these examples, people clearly know what they want - e.g., more pleasure from drugs or from gambling - and they select a decision which is the "best" from this viewpoint.

On top of this well-known abnormal decision making, there are also many examples when a seemingly rational decision is actually irrational. It is well known that people make seemingly irrational decisions as described above, i.e., that they are optimizing objective functions which lead to their physical and ethical ruin.

Somewhat less known for the general public - but well known in psychology is the fact that many quite rational people, people who are not addicted to drugs or gambling, people who normally lead a reasonably rational life, often make decisions which, at first glance, may seem reasonable but which, on deeper analysis, are irrational. This was first discovered in the studies of the Nobel Prize Winner Daniel Kahneman and his coauthor Amos Tversky; see, e.g., [8].

What they discovered is that sometimes people behave in such a way that no optimization can explain. Let us give a simple example. When a person is given two alternatives $\mathrm{A}$ and $\mathrm{B}$, the person is usually able to conclude that, e.g., to him or her, $\mathrm{A}$ is better than $\mathrm{B}$. We may disagree - as is the case of drug addiction - that $\mathrm{A}$ is an unhealthy choice, but A is what a person prefers. In this case, if now we offer the same person yet another alternative $\mathrm{C}$, he or she may stick to $\mathrm{A}$, or he or she may switch to $\mathrm{C}-$ but we do not expect this person to select $\mathrm{B}$ (since we already know that to this person, B is worse than another available alternative - namely, the alternative A). We do not expect to observe such a weird choice - but in some situations, this is exactly what has been observed; an example will be given later in this section.

This is a relatively clear example of a seemingly irrational decision making; we will show later that there are more subtle ones, where irrationality is not as easy to explain - but is clearly present; see examples in the next section.

So why irrational decision making? The fact that normal, reasonable people often make seemingly irrational decisions is puzzling. We humans come from billions years of improving evolution, we have flown to the Moon, we have discovered secrets of the Universe, we have learned to predict weather and to cure previously fatal diseases - this victorious image of Human Beings with a capital $\mathrm{H}$ does not seem to fit with simple decision mistakes, when the same person prefers A to B and then B to $\mathrm{A}$, without even realizing that he/she is inconsistent.

Yes, we are not perfect, there are wars, crimes, and exploitations, but the common wisdom seems to indicate that most of our problems are caused by our selfishness a criminal robs people because he wants to maximize his gain and he does not care if other people get hurt; a manufacturer of unhealthy foods maximizes his profit and does not care that people's health deteriorates as a result, etc. In all these cases, we 
blame the "evil" person for his selfish and vicious preferences, implicitly assuming that this person looks for what is best for him/her.

We can understand a person following a bad-for-society optimization criterion, but it is difficult to perceive a person whose decision making does not follow any optimization at all - and often, such a person is us.

How are such examples of seemingly irrational decision making explained now: the idea of bounded rationality. An established explanation for the seemingly irrational decision making is that we humans have a limited ability to process information - especially when the decision needs to be made urgently. On the qualitative level, this idea of bounded rationality is in good accordance with the observations; for example, usually, the more time we are given to make decisions, the more rational our decisions become; see, e.g., [8].

The existing explanations explain the very fact of seemingly irrational decision making, but not its observed specifics. The main limitation of the existing explanation is that it explains the fact that our decisions are sometimes not rational. In principle, under bounded resources, we can make different decisions, so we should observe various types of seemingly irrational decision making.

In many situations, however - for example, in the two situations described above - different decision makers exhibit the same deviations from the rational decision making. How can we explain these consistent deviations?

Main idea behind our explanations: optimization under granularity. When we do not have enough time to take all the information into account, a natural idea is to use partial information. For example, when a man sees an animal in the jungle, it could be a predator, so an immediate decision needs to be made on whether to run away or not. Ideally, we should take into account all the details of an animal image, but there is no time for that, a reasonable reaction is to run way if an animal is sufficiently large.

So, instead of considering each data set separately, we, in effect, combine these data sets into "granules" corresponding to the partial information that is actually used in decision making; see, e.g., [22]. In the above example, instead of using the animal's size, we only take into account whether this size is greater than a certain threshold $s_{0}$ or not. In effect, this means that we divide the set of all possible values of size into two granules:

- a granule consisting of small animals, whose size is smaller than $s_{0}$, and

- a granule consisting of large (and thus, potentially dangerous) animals, whose size is larger than or equal to $s_{0}$.

What we plan to show is that in many cases, if we take into account only algorithms that process such granular information, then the observed human decision making can be shown to be optimal among such granular algorithms - although, of course, if we could take into account all available information, we would be able to make a better decision. 
Comment. Some results from this paper were first presented at major fuzzy conferences $[12,13,14]$; several other results appear in this chapter for the first time.

Structure of the paper. Section 2 provides detailed description of two examples of seemingly irrational decision making. In Section 3, we show that granularity helps explain the first of these examples; the second example is explained in Section 4. Sections 5 and 6 show that similar granularity-based arguments can also explain why utility grows as square root of money (Section 5) and how fuzzy techniques can be reconciled with the traditional decision making (Section 6). Section 7 contains conclusions and future work.

\section{Examples of (Seemingly) Irrational Decision Making}

Examples of irrational decision making. Kahneman's book Thinking, Fast and Slow [8] has many examples of seemingly irrational decision making. In this chapter, we will concentrate on two examples. We selected these examples because they are, in our opinion, the easiest to explain without getting into the details of decision making theory and mathematical optimization. Let us describe these two examples in detail.

First example of seemingly irrational decision making: compromise effect. The first example comes from simple decisions that most of us do very frequently: decisions on what to buy.

A customer shopping for an item usually has several choices. Some of these choices have better quality, leading to more possibilities, etc. - but are, on the other hand, more expensive. For example, a customer shopping for a photo camera has plenty of choices ranging from the cheapest ones (that work only in good lighting) to professional cameras that enable the users to make highest-quality photos even under complex circumstances. A traveller planning to spend a night in a new city has a choice from the cheapest motels (which provide a place to sleep) to luxurious hotels providing all kinds of comfort, etc. A customer selects one of the alternatives by taking into account the additional advantages of more expensive choices versus the need to pay more money for these choices.

In many real-life situations, customers face numerous choices. As usual in science, a good way to understand complex phenomena is to start by analyzing the simplest cases. In line with this reasoning, researchers provided customers with two alternatives and recorded which of these two alternatives a customer selected. In many particular cases, these experiments helped better understand the customer's selections - and sometimes even predict customer selections.

At first glance, it seems like such pair-wise comparisons are all we need to know: if a customer faces several choices $a_{1}, a_{2}, \ldots, a_{n}$, then a customer will select an alternative $a_{i}$ if and only if this alternative is better in pair-wise comparisons that all other possible choices. To confirm this common-sense idea, in the 1990s, several 
researchers asked the customers to select one of the three randomly selected alternatives.

The experimenters expected that since the three alternatives were selected at random, a customers would:

- sometimes select the cheapest of the three alternative (of lowest quality of all three),

- sometimes select the intermediate alternative (or intermediate quality), and

- sometimes select the most expensive of the three alternatives (of highest quality of all three).

Contrary to the expectations, the experimenters observed that in the overwhelming majority of cases, customers selected the intermediate alternative; see, e.g., $[25,26,29]$. In all these cases, the customer selected an alternative which provided a compromise between the quality and cost; because of this, this phenomenon was named compromise effect.

Why is this irrational? At first glance, selecting the middle alternative is reasonable. Let us show, however, that such a selection is not always rational.

For example, let us assume that we have four alternative $a_{1}<a_{2}<a_{3}<a_{4}$ ordered in the increasing order of price and at the same time, increasing order of quality. Then:

- if we present the user with three choices $a_{1}<a_{2}<a_{3}$, in most cases, the user will select the middle choice $a_{2}$; this means, in particular, that, to the user, $a_{2}$ better than the alternative $a_{3}$;

- on the other hand, if we present the user with three other choices $a_{2}<a_{3}<a_{4}$, in most cases, the same user will select the middle choice $a_{3}$; but this means that, to the user, the alternative $a_{3}$ better than the alternative $a_{2}$.

If in a pair-wise comparison, $a_{2}$ is better, then the second choice is wrong. If in a pair-wise comparison, the alternative $a_{3}$ is better, then the first choice is wrong. In both cases, one of the two choices is irrational.

This is not just an experimental curiosity, customers' decisions have been manipulated this way. At first glance, the above phenomena may seem like one of optical illusions or logical paradoxes: interesting but not that critically important. Actually, it is serious and important, since, according to anecdotal evidence, many companies have tried to use this phenomenon to manipulate the customer's choices: to make the customer buy a more expensive product.

For example, if there are two possible types of a certain product, a company can make sure that most customers select the most expensive type - simply by offering, as the third option, an even more expensive type of the same product.

Manipulation possibility has been exaggerated, but mystery remains. Recent research showed that manipulation is not very easy: the compromise effect only happens when a customer has no additional information - and no time (or no desire) to collect such information. In situations when customers were given access to additional information, they selected - as expected from rational folks - one of the 
three alternatives with almost equal frequency, and their pairwise selections, in most cases, did not depend on the presence of any other alternatives; see, e.g., [28].

The new experiment shows that the compromise effect is not as critical and not as wide-spread as it was previously believed. However, in situation when decisions need to be made under major uncertainty, this effect is clearly present - and its seemingly counterintuitive, inconsistent nature is puzzling.

Second example of seemingly irrational decision making: biased probability estimates. In the first example, we considered situations with a simple choice, in which we have several alternatives, and we know the exact consequences of each alternative. In many practical cases, the situation is more complicated: for each decision, depending on how things go, we may face different consequences. For example, if a person invests all his retirement money in the stock market, the market may go up - in which case, he will gain - or it may go down, in which case he will lose a big portion of his savings. A person who takes on a potentially dangerous sport (like car racing) will probably gain a lot of pleasure, but there is also a chance of a serious injury.

To make a decision in such situations, it is important to estimate the probability of different outcomes. In some cases we know these probabilities - e.g., in the staterun lotteries, probabilities of winning are usually disclosed. In other cases, a person has to estimate these probabilities.

Of course, based on the limited information, we can get only approximate estimates of the corresponding probabilities. However, we expect that these estimates are unbiased, i.e., that, on average, they should provide a reasonably accurate estimate. Indeed, if we systematically overestimate small probabilities, then we would overestimate our gain a lottery and, on average, lose. Similarly, if we systematically underestimate small probabilities, then, in particular, we will underestimate the probability of a disaster and invest in too risky stocks - and also lose on average.

This is what we expect: unbiased estimates, but this is not what we observe. What we observe is that small probabilities are routinely overestimated, while probabilities close to 1 are routinely underestimated. This is not just an occasional phenomenon: for each actual probability, the estimated probability is consistently different. For different actual probabilities $p_{i}$, the corresponding estimated probabilities $\widetilde{p}_{i}$ are given in [8] (see also references therein):

\begin{tabular}{|l||c|c|c|c|c|c|c|c|c|c|c|c|c|}
\hline$p_{i}$ & 0 & 1 & 2 & 5 & 10 & 20 & 50 & 80 & 90 & 95 & 98 & 99 & 100 \\
\hline$\widetilde{p}_{i}$ & 0 & 5.5 & 8.1 & 13.2 & 18.6 & 26.1 & 42.1 & 60.1 & 71.2 & 79.3 & 87.1 & 91.2 & 100 \\
\hline
\end{tabular}

Why biased estimates? As we have mentioned, biased estimates are contrary to rational decision making: overestimating a small probability of success may get a decision maker involved in risky situations where, on average (and thus, in the long run) the decision maker will lose. On the other hand, overestimating a small probability of a disaster will make a decision maker too cautious and prevent him/her from making a rational risky decision. 


\section{Explaining the First Example of Seemingly Irrational Human Decision making: Granularity Explains the Compromise Effect}

Compromise effect: reminder. We have three alternative $a, a^{\prime}$ and $a^{\prime \prime}$ :

- the alternative $a$ is the cheapest - and is, correspondingly, of the lowest quality among the given three alternatives;

- the alternative $a^{\prime}$ is intermediate in terms of price - and is, correspondingly, intermediate in terms of quality;

- finally, the alternative $a^{\prime \prime}$ is the most expensive - and is, correspondingly, of the highest quality among the given three alternatives.

We do not know the exact prices, we just know the order between them; similarly, we do not know the exact values of quality, we just know the order between them. In this situation, most people select an alternative $a^{\prime}$.

Let us describe the corresponding granularity. The "utility" of each alternative comes from two factors:

- the first factor comes from the quality: the higher the quality, the better - i.e., larger the corresponding component $u_{1}$ of the utility;

- the second factor comes from price: the lower the price, the better for the user i.e., the larger the corresponding component $u_{2}$ of the utility.

The fact that we do not know the exact value of the price means, in effect, that we consider three possible levels of price and thus, three possible levels of the utility $u_{1}$ :

- low price, corresponding to high price-related utility;

- medium price, corresponding to medium price-related utility; and

- high price, corresponding to low price-related utility.

In the following text, we will denote "low" by $L$, "medium" by $M$, and "high" by $H$. In these terms, the above description of each alternative by the corresponding pair of utility values takes the following form:

- the alternative $a$ is characterized by the pair $(L, H)$;

- the alternative $a^{\prime}$ is characterized by the pair $(M, M)$; and

- the alternative $a^{\prime \prime}$ is characterized by the pair $(H, L)$.

Natural symmetries. We do not know a priori which of the two utility components is more important. As a result, it is reasonable to treat both components equally. In order words, the selection should be the same if we simply swap the two utility components - i.e., we should select the same of three alternatives before and after swap:

- if we are selecting an alternative based on the pairs $(L, H),(M, M)$, and $(H, L)$,

- then we should select the exact same alternative if the pairs were swapped, i.e., if: 
- the alternative $a$ was characterized by the pair $(H, L)$;

- the alternative $a^{\prime}$ was characterized by the pair $(M, M)$; and

- the alternative $a^{\prime \prime}$ was characterized by the pair $(L, H)$.

Similarly, there is no reason to a priori prefer one alternative or the other. So, the selection should not depend on which of the alternatives we mark as $a$, which we mark as $a^{\prime}$, and which we mark as $a^{\prime \prime}$. In other words, any permutation of the three alternatives is a reasonable symmetry transformation. For example, if, in our case, we select an alternative $a$ which is characterized by the pair $(L, H)$, then, after we swap $a$ and $a^{\prime \prime}$ and get the choice of the following three alternatives:

- the alternative $a$ which is characterized by the pair $(H, L)$;

- the alternative $a^{\prime}$ is characterized by the pair $(M, M)$; and

- the alternative $a^{\prime \prime}$ is characterized by the pair $(L, H)$,

then we should select the same alternative - which is now denoted by $a^{\prime \prime}$.

What can be conclude based on these symmetries. Now, we can observe the following: that if we both swap $u_{1}$ and $u_{2}$ and swap $a$ and $a^{\prime \prime}$, then you get the exact same characterization of all alternatives:

- the alternative $a$ is still characterized by the pair $(L, H)$;

- the alternative $a^{\prime}$ is still characterized by the pair $(M, M)$; and

- the alternative $a^{\prime \prime}$ is still characterized by the pair $(H, L)$.

The only difference is that:

- now, $a$ indicates an alternative which was previously denoted by $a^{\prime \prime}$, and

- $a^{\prime \prime}$ now denotes the alternative which was previously denoted by $a$.

As we have mentioned, it is reasonable to conclude that:

- if in the original triple selection, we select the alternative $a$,

- then in the new selection - which is based on the exact same pairs of utility values - we should also select an alternative denoted by $a$.

But this "new" alternative $a$ is nothing else but the old $a^{\prime \prime}$. So, we conclude that:

- if we selected $a$,

- then we should have selected a different alternative $a^{\prime \prime}$ in the original problem.

This is clearly a contradiction:

- we started by assuming that, to the user $a$ was better than $a^{\prime \prime}$ (because otherwise $a$ would not have been selected in the first place), and

- we ended up concluding that to the same user, the original alternative $a^{\prime \prime}$ is better than $a$.

This contradiction shows that, under the symmetry approach, we cannot prefer $a$. Similarly:

- if in the original problem, we preferred an alternative $a^{\prime \prime}$, 
- then this would mean that in the new problem, we should still select an alternative which marked by $a^{\prime \prime}$.

But this "new" $a$ " is nothing else but the old $a$. So, this means that:

- if we originally selected $a^{\prime \prime}$,

- then we should have selected a different alternative $a$ in the original problem.

This is also a contradiction:

- we started by assuming that, to the user $a^{\prime \prime}$ was better than $a$ (because otherwise $a^{\prime \prime}$ would not have been selected in the first place), and

- we ended up concluding that to the same user, the original alternative $a$ is better than $a^{\prime \prime}$. This contradiction shows that, under the symmetry approach, we cannot prefer $a^{\prime \prime}$.

We thus conclude that out of the three alternatives $a, a^{\prime}$, and $a^{\prime \prime}$ :

- we cannot select $a$, and

- we cannot select $a^{\prime \prime}$.

This leaves us only once choice: to select the intermediate alternative $a^{\prime}$.

This is exactly the compromise effect that we planned to explain.

Conclusion. Experiments show when people are presented with three choices $a<$ $a^{\prime}<a^{\prime \prime}$ of increasing price and increasing quality, and they do not have detailed information about these choices, then in the overwhelming majority of cases, they select the intermediate alternative $a^{\prime}$.

This "compromise effect" is, at first glance, irrational: selecting $a^{\prime}$ means that, to the user, $a^{\prime}$ is better than $a^{\prime \prime}$, but in a similar situation when the user is presented with $a^{\prime}<a^{\prime \prime}<a^{\prime \prime \prime}$, the same principle would indicate that the user will select $a^{\prime \prime}-$ meaning that $a^{\prime \prime}$ is better than $a^{\prime}$.

Somewhat surprisingly, a natural granularity approach explains this seemingly irrational decision making.

\section{Explaining the Second Example of Seemingly Irrational Human Decision making: Granularity Explains Why Our Probability Estimates Are Biased}

Main idea. Probability of an event is estimated, from observations, as the frequency with which this event occurs. For example, if out of 100 days of observation, rain occurred in 40 of these days, then we estimate the probability of rain as $40 \%$. In general, if out of $n$ observations, the event was observed in $k$ of them, we estimate the probability as the ratio $\frac{k}{n}$.

This ratio is, in general, different from the actual (unknown) probability. For example, if we take a fair coin, for which the probability of head is exactly $50 \%$, and 
flip it 100 times, we may get 50 heads, but we may also get 47 heads, 52 heads, etc. Similarly, if we have the coin fall heads 50 times out of 100, the actual probability could be $50 \%$, could be $47 \%$ and could be $52 \%$. In other words, instead of the exact value of the probability, we get a granule of possible values. (In statistics, this granule is known as a confidence interval; see, e.g., [27].)

In other words:

- first, we estimate a probability based on the observations; as a result, instead of the exact value, we get a granule which contains the actual (unknown) probability; this granule is all we know about the actual probability;

- then, when a need comes to estimate the probability, we produce an estimate based on the granule.

Let us analyze these two procedures one by one.

Probability granules: analysis of the first procedure and the resulting formulas. It is known (see, e.g., [27]), that the expected value of the frequency is equal to $p$, and that the standard deviation of this frequency is equal to

$$
\sigma=\sqrt{\frac{p \cdot(1-p)}{n}} .
$$

It is also known that, due to the Central Limit Theorem, for large $n$, the distribution of frequency is very close to the normal distribution (with the corresponding mean $p$ and standard deviation $\sigma$ ).

For normal distribution, we know that with a high certainty all the values are located within 2-3 standard deviations from the mean, i.e., in our case, within the interval $\left(p-k_{0} \cdot \sigma, p+k_{0} \cdot \sigma\right)$, where $k_{0}=2$ or $k_{0}=3$ : for example, for $k_{0}=3$, this is true with confidence $99.9 \%$. We can thus say that the two values of probability $p$ and $p^{\prime}$ are (definitely) distinguishable if the corresponding intervals of possible values of frequency do not intersect - and thus, we can distinguish between these two probabilities just by observing the corresponding frequencies.

In precise terms, the probabilities $p<p^{\prime}$ are distinguishable if

$$
\left(p-k_{0} \cdot \sigma, p+k_{0} \cdot \sigma\right) \cap\left(p^{\prime}-k_{0} \cdot \sigma^{\prime}, p+k_{0} \cdot \sigma^{\prime}\right)=\emptyset,
$$

where

$$
\sigma^{\prime} \stackrel{\text { def }}{=} \sqrt{\frac{p^{\prime} \cdot\left(1-p^{\prime}\right)}{n}},
$$

i.e., if $p^{\prime}-k_{0} \cdot \sigma^{\prime} \geq p+k_{0} \cdot \sigma$. The smaller $p^{\prime}$, the smaller the difference $p^{\prime}-k_{0} \cdot \sigma^{\prime}$. Thus, for a given probability $p$, the next distinguishable value $p^{\prime}$ is the one for which

$$
p^{\prime}-k_{0} \cdot \sigma^{\prime}=p+k_{0} \cdot \sigma .
$$

When $n$ is large, these value $p$ and $p^{\prime}$ are close to each other; therefore, $\sigma^{\prime} \approx$ $\sigma$. Substituting an approximate value $\sigma$ instead of $\sigma^{\prime}$ into the above equality, we conclude that 


$$
p^{\prime} \approx p+2 k_{0} \cdot \sigma=p+2 k_{0} \cdot \sqrt{\frac{p \cdot(1-p)}{n}} .
$$

If the value $p$ corresponds to the $i$-th level, then the next value $p^{\prime}$ corresponds to the $(i+1)$-st level. Let us denote the value corresponding to the $i$-th level by $p(i)$. In these terms, the above formula takes the form

$$
p(i+1)-p(i)=2 k_{0} \cdot \sqrt{\frac{p \cdot(1-p)}{n}} .
$$

The above notation defines the value $p(i)$ for non-negative integers $i$. We can extrapolate this dependence so that it will be defined for all non-negative real values $i$.

When $n$ is large, the values $p(i+1)$ and $p(i)$ are close, the difference

$$
p(i+1)-p(i)
$$

is small, and therefore, we can expand the expression $p(i+1)$ in Taylor series and keep only linear terms in this expansion:

$$
p(i+1)-p(i) \approx \frac{d p}{d i} .
$$

Substituting the above expression for $p(i+1)-p(i)$ into this formula, we conclude that

$$
\frac{d p}{d i}=\mathrm{const} \cdot \sqrt{p \cdot(1-p)} .
$$

Moving all the terms containing $p$ into the left-hand side and all the terms containing $i$ into the right-hand side, we get

$$
\frac{d p}{\sqrt{p \cdot(1-p)}}=\text { const } \cdot d i
$$

Integrating this expression and taking into account that $p=0$ corresponds to the lowest 0 -th level -i.e., that $i(0)=0$ - we conclude that

$$
i(p)=\text { const } \cdot \int_{0}^{p} \frac{d q}{\sqrt{q \cdot(1-q)}} .
$$

This integral can be easily computed if introduce a new variable $t$ for which $q=$ $\sin ^{2}(t)$. In this case,

$$
d q=2 \cdot \sin (t) \cdot \cos (t) \cdot d t
$$

$1-p=1-\sin ^{2}(t)=\cos ^{2}(t)$ and therefore,

$$
\sqrt{p \cdot(1-p)}=\sqrt{\sin ^{2}(t) \cdot \cos ^{2}(t)}=\sin (t) \cdot \cos (t) .
$$


The lower bound $q=0$ corresponds to $t=0$ and the upper bound $q=p$ corresponds to the value $t_{0}$ for which $\sin ^{2}\left(t_{0}\right)=p$ - i.e., $\sin \left(t_{0}\right)=\sqrt{p}$ and $t_{0}=\arcsin (\sqrt{p})$. Therefore,

$$
\begin{gathered}
i(p)=\text { const } \cdot \int_{0}^{p} \frac{d q}{\sqrt{q \cdot(1-q)}}=\text { const } \cdot \int_{0}^{t_{0}} \frac{2 \cdot \sin (t) \cdot \cos (t) \cdot d t}{\sin (t) \cdot \cos (t)}= \\
\int_{0}^{t_{0}} 2 \cdot d t=2 \cdot \text { const } \cdot t_{0} .
\end{gathered}
$$

We know how $t_{0}$ depends on $p$, so we get

$$
i(p)=2 \cdot \text { const } \cdot \arcsin (\sqrt{p}) .
$$

We can determine the constant from the condition that the largest possible probability value $p=1$ should correspond to the largest level $i=m$. From the condition that $i(1)=m$, taking into account that

$$
\arcsin (\sqrt{1})=\arcsin (1)=\frac{\pi}{2},
$$

we conclude that

$$
i(p)=\frac{2 m}{\pi} \cdot \arcsin (\sqrt{p}) .
$$

Thus,

$$
\arcsin (\sqrt{p})=\frac{\pi \cdot i}{2 m},
$$

hence

$$
\sqrt{p}=\sin \left(\frac{\pi \cdot i}{2 m}\right)
$$

and thus,

$$
p(i)=\sin ^{2}\left(\frac{\pi \cdot i}{2 m}\right) .
$$

Thus, probability granules are formed by intervals $[p(i), p(i+1)]$. Each empirical probability is represented by the granule $i$ to which is belongs.

From granules to probability estimates: analysis of the second procedure. As we have mentioned, instead of the actual probabilities, we have probability labels corresponding to $m$ different granules:

- the first label corresponds to the smallest certainty,

- the second label corresponds to the second smallest certainty,

- etc.,

- until we reach the last label which corresponds to the largest certainty.

We need to produce some estimates of the probability based on the granule. In other words, for each $i$ from 1 to $m$, we need to assign, to each $i$-th label, a value $p_{i}$ in 
such a way that labels corresponding to higher certainty should get larger numbers: $p_{1}<p_{2}<\ldots<p_{m}$.

Before we analyze how to do it, let us recall that one of the main objectives of assigning numerical values is that we want computers to help us solve the corresponding decision problems, and computers are not very good in dealing with granules; their natural language is the language of numbers. From this viewpoint, it makes sense to consider not all theoretically possible exact real numbers, but only computer-representable real numbers.

In a computer, real numbers from the interval $[0,1]$ are usually represented by the first $d$ digits of their binary expansion. Thus, computer-representable numbers are $0, h \stackrel{\text { def }}{=} 2^{-d}, 2 h, 3 h, \ldots$, until we reach the value $2^{d} \cdot h=1$.

In our analysis, we will assume that the "machine unit" $h>0$ is fixed, and we will this assume that only multiples of this machine units are possible values of all $n$ probabilities $p_{i}$.

For example, when $h=0.1$, each probability $p_{i}$ takes 11 possible values: $0,0.1$, $0.2,0.3,0.4,0.5,0.6,0.7,0.8,0.9$, and 1.0 .

In the modern computers, the value $h$ is extremely small; thus, whenever necessary, we can assume that $h \approx 0$ - i.e., use limit case of $h \rightarrow 0$ instead of the actual small "machine unit" $h$.

For each $h$, we consider all possible combinations of probabilities $p_{1}<\ldots<p_{m}$ in which all the numbers $p_{i}$ are proportional to the selected step $h$, i.e., all possible combinations of values $\left(k_{1} \cdot h, \ldots, k_{m} \cdot h\right)$ with $k_{1}<\ldots<k_{m}$.

For example, when $m=2$ and $h=0.1$, we consider all possible combinations of values $\left(k_{1} \cdot h, k_{2} \cdot h\right)$ with $k_{1}<k_{2}$ :

- For $k_{1}=0$ and $p_{1}=0$, we have 10 possible combinations $(0,0.1),(0,0.2), \ldots$, $(0,1)$.

- For $k_{1}=1$ and $p_{1}=0.1$, we have 9 possible combinations $(0.1,0.2),(0.1,0.3)$, $\ldots,(0.1,1)$.

- ...

- Finally, for $k_{1}=9$ and $p_{1}=0.9$, we have only one possible combination $(0.9,1)$.

For each $i$, for different possible combinations $\left(p_{1}, \ldots, p_{m}\right)$, we get, in general, different value of the probability $p_{i}$. According to the complete probability formula, we can obtain the actual (desired) probability $P_{i}$ if we combine all these value $p_{i}$ with the weights proportional to the probabilities of corresponding combinations:

$$
P_{i}=\sum_{p_{1}<\ldots<p_{m}} p_{i} \cdot \operatorname{Prob}\left(p_{1}, \ldots, p_{m}\right) .
$$

Since we have no reason to believe that some combinations $\left(p_{1}, \ldots, p_{m}\right)$ are more probable and some are less probable, it is thus reasonable to assume that all these combinations are equally probable. Thus, $P_{i}$ is equal to the arithmetic average of the values $p_{i}$ corresponding to all possible combinations $\left(p_{1}, \ldots, p_{m}\right)$.

For example, for $m=2$ and $h=0.1$, we thus estimate $P_{1}$ by taking an arithmetic average of the values $p_{1}$ corresponding to all possible pairs. Specifically, we average: 
- ten values $p_{1}=0$ corresponding to ten pairs $(0,0.1), \ldots,(0,1)$;

- nine values $p_{1}=0.1$ corresponding to nine pairs $(0.1,0.2), \ldots,(0.1,1)$;

- ...

- and a single value $p_{1}=0.9$ corresponding to the single pair $(0.9,1)$.

As a result, we get the value

$$
P_{1}=\frac{10 \cdot 0.0+0 \cdot 0.1+\ldots+1 \cdot 0.9}{10+9+\ldots+1}=\frac{16.5}{55}=0.3 .
$$

Similarly, to get the value $p_{2}$, we average:

- a single value $p_{2}=0.1$ corresponding to the single pair $(0,0.1)$;

- two values $p_{2}=0.2$ corresponding to two pairs $(0,0.2)$ and $(0.1,0.2)$;

- ...

- ten values $p_{2}=1.0$ corresponding to ten pairs $(0,1), \ldots,(0.9,1)$.

As a result, we get the value

$$
P_{2}=\frac{1 \cdot 0.1+2 \cdot 0.2+\ldots+10 \cdot 1.0}{1+2+\ldots+10}=\frac{37.5}{55}=0.7 .
$$

The probability $p_{i}$ of each label can take any of the equidistant values $0, h, 2 h, 3 h$, $\ldots$, with equal probability. In the limit $h \rightarrow 0$, the resulting probability distribution tends to the uniform distribution on the interval $[0,1]$.

In this limit $h \rightarrow 0$, we get the following problem:

- we start with $m$ independent random variable $v_{1}, \ldots, v_{m}$ which are uniformly distributed on the interval $[0,1]$;

- we then need to find, for each $i$, the conditional expected value

$$
E\left[v_{i} \mid v_{1}<\ldots<v_{m}\right]
$$

of each variable $v_{i}$ under the condition that the values $v_{i}$ are sorted in increasing order.

Conditional expected values are usually more difficult to compute than unconditional ones. So, to solve our problem, let us reduce our problem to the problem of computing the unconditional expectation.

Let us consider $m$ independent random variables each of which is uniformly distributed on the interval $[0,1]$. One can easily check that for any two such variables $v_{i}$ and $v_{j}$, the probability that they are equal to each other is 0 . Thus, without losing generality, we can safely assume that all $m$ random values are different. Therefore, the whole range $[0,1]^{m}$ is divided into $m$ ! sub-ranges corresponding to different orders between $v_{i}$. Each sub-range can be reduced to the sub-range corresponding to $v_{1}<\ldots<v_{m}$ by an appropriate permutation in which $v_{1}$ is swapped with the smallest $v_{(1)}$ of $m$ values, $v_{2}$ is swapped with the second smallest $v_{(2)}$, etc.

Thus, the conditional expected value of $v_{i}$ is equal to the (unconditional) expected value of the $i$-th value $v_{(i)}$ in the increasing order. This value $v_{(i)}$ is known as an order 
statistic, and for uniform distributions, the expected values of all order statistics are known (see, e.g., $[1,2,5]): P_{i}=\frac{i}{m+1}$.

So, if all we know is that our degree of certainty is expressed by $i$-th label on an $m$-label scale of granules, then it is reasonable to assign, to this case, the probability $P_{i}=\frac{i}{m+1}$.

Let us now combine the two procedures. In the first procedure, based on the empirical frequency $p$, we find a label $i$ for which

$$
p \approx \sin ^{2}\left(\frac{\pi \cdot i}{2 m}\right)
$$

Based on this label, we then estimate the probability as $P_{i}=\frac{i}{m+1}$. For large $m$, we have $P \approx \frac{i}{m}$. Substituting $P$ instead of $\frac{i}{m}$ into the formula for $p$, we conclude that

$$
p \approx \sin ^{2}\left(\frac{\pi}{2} \cdot P\right)
$$

Based on this formula, we can express the estimate $P$ in terms of the actual probability $p$, as

$$
P \approx \frac{1}{\pi} \cdot \arcsin (\sqrt{p})
$$

Comparing our estimates $P$ with empirical probability estimates $\widetilde{p}_{i}$ : first try. Let us compare the probabilities $p_{i}$, Kahneman's empirical estimates $\widetilde{p}_{i}$, and the estimates $P_{i}=\frac{1}{\pi} \cdot \arcsin \left(\sqrt{p_{i}}\right)$ computed by using the above formula:

\begin{tabular}{|c||c|c|c|c|c|c|c|c|c|c|c|c|c|}
\hline$p_{i}$ & 0 & 1 & 2 & 5 & 10 & 20 & 50 & 80 & 90 & 95 & 98 & 99 & 100 \\
\hline$\widetilde{p}_{i}$ & 0 & 5.5 & 8.1 & 13.2 & 18.6 & 26.1 & 42.1 & 60.1 & 71.2 & 79.3 & 87.1 & 91.2 & 100 \\
\hline$P_{i}$ & 0 & 6.4 & 9.0 & 14.4 & 20.5 & 29.5 & 50.0 & 70.5 & 79.5 & 85.6 & 91.0 & 93.6 & 100 \\
\hline
\end{tabular}

The estimates $P_{i}$ are closer to the empirical probability estimates $\widetilde{p}_{i}$ than the original probabilities, but the relation does not seem very impressive.

We will show that the fit is much better than it seems at first glance. At first glance, the above direct comparison between the observed estimates $\widetilde{p}_{i}$ and the values $P_{i}$ seems to make perfect sense. However, let us look deeper.

The observed estimates come from the fact that users select an alternative $a$ that maximizes the expected gain $u(a)=\sum w_{i}(a) \cdot u_{i}$, where $w_{i}(a) \stackrel{\text { def }}{=} p_{i}(a)$. It is easy to observe that if we multiply all the weights by the same positive constant $\lambda>0$, i.e., consider the weights $w_{i}^{\prime}(a)=\lambda \cdot w_{i}(a)$, then for each action, the resulting value of the weighted gain will also increase by the same factor: 


$$
w^{\prime}(a)=\sum w_{i}^{\prime}(a) \cdot u_{i}=\sum \lambda \cdot w_{i}(a) \cdot u_{i}=\lambda \cdot \sum w_{i}(a) \cdot u_{i}=\lambda \cdot w_{i}(a) .
$$

The relation between the weighted gains of two actions $a$ and $a^{\prime}$ does not change if we simply multiply both gains by a positive constant:

- if $w_{i}(a)<w_{i}\left(a^{\prime}\right)$, then, multiplying both sides of this inequality by $\lambda$, we get $w_{i}^{\prime}(a)<w_{i}^{\prime}\left(a^{\prime}\right)$

- if $w_{i}(a)=w_{i}\left(a^{\prime}\right)$, then, multiplying both sides of this equality by $\lambda$, we get $w_{i}^{\prime}(a)=w_{i}^{\prime}\left(a^{\prime}\right)$

- if $w_{i}(a)>w_{i}\left(a^{\prime}\right)$, then, multiplying both sides of this inequality by $\lambda$, we get $w_{i}^{\prime}(a)>w_{i}^{\prime}\left(a^{\prime}\right)$.

All we observe is which of the two actions a person selects. Since multiplying all the weights by a constant does not change the selection, this means that based on the selection, we cannot uniquely determine the weights: an empirical selection which is consistent with the weights $w_{i}$ is equally consistent with the weights $w_{i}^{\prime}=\lambda \cdot w_{i}$.

This fact can be used to normalize the empirical weights, i.e., to multiply them by a constant so as to satisfy some additional condition.

In [8], to normalize the weights, the authors use the requirement that the weight corresponding to probability 1 should be equal to 1 . Since for $p=1$, the corresponding value $P$ is also equal to 1 , we get a perfect match for $p=1$, but a rather lousy match for probabilities intermediate between 0 and 1 .

Instead of this normalization, we can select $\lambda$ so as to get the best match "on average".

How to improve the fit: details. A natural idea is to select $\lambda$ from the Least Squares method, i.e., select $\lambda$ for which the relative mean squares difference

$$
\sum_{i}\left(\frac{\lambda \cdot P_{i}-\widetilde{p}_{i}}{P_{i}}\right)^{2}
$$

is the smallest possible. Differentiating this expression with respect to $\lambda$ and equating the derivative to 0 , we conclude that

$$
\sum_{i}\left(\lambda-\frac{\widetilde{p}_{i}}{P_{i}}\right)=0
$$

i.e., that

$$
\lambda=\frac{1}{m} \cdot \sum_{i} \frac{\widetilde{p}_{i}}{P_{i}}
$$

Resulting match. For the above values, this formula leads to $\lambda=0.910$.

Result. The resulting values $P_{i}^{\prime}=\lambda \cdot P_{i}$ are much closer to the empirical probabilities $\widetilde{p}_{i}$ : 


\begin{tabular}{|c||c|c|c|c|c|c|c|c|c|c|c|c|c|}
\hline$p_{i}$ & 0 & 1 & 2 & 5 & 10 & 20 & 50 & 80 & 90 & 95 & 98 & 99 & 100 \\
\hline$\widetilde{p}_{i}$ & 0 & 5.5 & 8.1 & 13.2 & 18.6 & 26.1 & 42.1 & 60.1 & 71.2 & 79.3 & 87.1 & 91.2 & 100 \\
\hline$P_{i}^{\prime}=\lambda \cdot P_{i}$ & 0 & 5.8 & 8.2 & 13.1 & 18.7 & 26.8 & 45.5 & 64.2 & 72.3 & 77.9 & 82.8 & 87.4 & 91.0 \\
\hline
\end{tabular}

For most probabilities $p_{i}$, the difference between the values $P_{i}^{\prime}$ and the empirical probability estimates $\widetilde{p}_{i}$ is so small that it is below the accuracy with which the empirical weights can be obtained from the experiment.

Thus, granularity ideas indeed explain Kahneman and Tversky's observation of biased empirical probability estimates.

Conclusion. Kahneman and Tversky showed that when people make decision, then instead of - as should be rational - weighting outcomes with weights proportional to probabilities of different outcomes - they use biased weights, overestimating the importance of low-probability events and underestimating the importance of highprobability events. In this section, we show that this observable bias can be explain if we take into account granularity - imposed by our limited rationality (i.e., our limited ability to process information).

\section{Granularity Explains Why Utility Grows as Square Root of Money}

What we do in this section. In this section, we provide another example when granularity explains observed decision making. To explain this example, we need to recall the traditional decision theory and the corresponding notion of utility.

Main assumption behind the traditional decision theory. Traditional approach to decision making is based on an assumption that for each two alternatives $A^{\prime}$ and $A^{\prime \prime}$, a user can tell:

- whether the first alternative is better for him/her; we will denote this by $A^{\prime \prime}<A^{\prime}$;

- or the second alternative is better; we will denote this by $A^{\prime}<A^{\prime \prime}$;

- or the two given alternatives are of equal value to the user; we will denote this by $A^{\prime}=A^{\prime \prime}$.

Towards a numerical description of preferences: the notion of utility. Under the above assumption, we can form a natural numerical scale for describing preferences. Namely, let us select a very bad alternative $A_{0}$ and a very good alternative $A_{1}$. Then, most other alternatives are better than $A_{0}$ but worse than $A_{1}$.

For every probability $p \in[0,1]$, we can form a lottery $L(p)$ in which we get $A_{1}$ with probability $p$ and $A_{0}$ with probability $1-p$.

- When $p=0$, this lottery coincides with the alternative $A_{0}: L(0)=A_{0}$.

- When $p=1$, this lottery coincides with the alternative $A_{1}: L(1)=A_{1}$. 
For values $p$ between 0 and 1 , the lottery is better than $A_{0}$ and worse than $A_{1}$. The larger the probability $p$ of the positive outcome increases, the better the result:

$$
p^{\prime}<p^{\prime \prime} \text { implies } L\left(p^{\prime}\right)<L\left(p^{\prime \prime}\right) .
$$

Thus, we have a continuous scale of alternatives $L(p)$ that monotonically goes from $L(0)=A_{0}$ to $L(1)=A_{1}$. We will use this scale to gauge the attractiveness of each alternative $A$.

Due to the above monotonicity, when $p$ increases, we first have $L(p)<A$, then we have $L(p)>A$, and there is a threshold separating values $p$ for which $L(p)<A$ from the values $p$ for which $L(p)>A$. This threshold value is called the utility of the alternative $A$ :

$$
u(A) \stackrel{\text { def }}{=} \sup \{p: L(p)<A\}=\inf \{p: L(p)>A\} .
$$

Then, for every $\varepsilon>0$, we have

$$
L(u(A)-\varepsilon)<A<L(u(A)+\varepsilon) .
$$

We will describe such (almost) equivalence by $\equiv$, i.e., we will write that $A \equiv$ $L(u(A))$.

How to elicit the utility from a user: a fast iterative process. Initially, we know the values $\underline{u}=0$ and $\bar{u}=1$ such that $A \equiv L(u(A))$ for some $u(A) \in[\underline{u}, \bar{u}]$.

On each stage of this iterative process, once we know values $\underline{u}$ and $\bar{u}$ for which $u(A) \in[\underline{u}, \bar{u}]$, we compute the midpoint $u_{\text {mid }}$ of the interval $[\underline{u}, \bar{u}]$ and ask the user to compare $A$ with the lottery $L\left(u_{\mathrm{mid}}\right)$ corresponding to this midpoint. There are two possible outcomes of this comparison: $A \leq L\left(u_{\mathrm{mid}}\right)$ and $L\left(u_{\mathrm{mid}}\right) \leq A$.

- In the first case, the comparison $A \leq L\left(u_{\text {mid }}\right)$ means that $u(A) \leq u_{\text {mid }}$, so we can conclude that $u \in\left[\underline{u}, u_{\text {mid }}\right]$.

- In the second case, the comparison $L\left(u_{\text {mid }}\right) \leq A$ means that $u_{\text {mid }} \leq u(A)$, so we can conclude that $u \in\left[u_{\text {mid }}, \bar{u}\right]$.

In both cases, after an iteration, we decrease the width of the interval $[u, \bar{u}]$ by half. So, after $k$ iterations, we get an interval of width $2^{-k}$ which contains $u(A)-$ i.e., we get $u(A)$ with accuracy $2^{-k}$.

How to make a decision based on utility values. Suppose that we have found the utilities $u\left(A^{\prime}\right), u\left(A^{\prime \prime}\right), \ldots$, of the alternatives $A^{\prime}, A^{\prime \prime}, \ldots$ Which of these alternatives should we choose?

By definition of utility, we have:

- $A \equiv L(u(A))$ for every alternative $A$, and

- $L\left(p^{\prime}\right)<L\left(p^{\prime \prime}\right)$ if and only if $p^{\prime}<p^{\prime \prime}$.

We can thus conclude that $A^{\prime}$ is preferable to $A^{\prime \prime}$ if and only if $u\left(A^{\prime}\right)>u\left(A^{\prime \prime}\right)$. In other words, we should always select an alternative with the largest possible value of utility. So, to find the best solution, we must solve the corresponding optimization problem. 
Before we go further: caution. We are not claiming that people estimate probabilities when they make decisions: we know they often don't. Our claim is that when people make definite and consistent choices, these choices can be described by probabilities. (Similarly, a falling rock does not solve equations but follows Newton's equations $m a=m \frac{d^{2} x}{d t^{2}}=-m g$.) In practice, decisions are often not definite (uncertain) and not consistent.

How to estimate utility of an action. For each action, we usually know possible outcomes $S_{1}, \ldots, S_{n}$. We can often estimate the probabilities $p_{1}, \ldots, p_{n}$ of these outcomes.

By definition of utility, each situation $S_{i}$ is equivalent to a lottery $L\left(u\left(S_{i}\right)\right)$ in which we get:

- $A_{1}$ with probability $u\left(S_{i}\right)$ and

- $A_{0}$ with the remaining probability $1-u\left(S_{i}\right)$.

Thus, the original action is equivalent to a complex lottery in which:

- first, we select one of the situations $S_{i}$ with probability $p_{i}: P\left(S_{i}\right)=p_{i}$;

- then, depending on $S_{i}$, we get $A_{1}$ with probability $P\left(A_{1} \mid S_{i}\right)=u\left(S_{i}\right)$ and $A_{0}$ with probability $1-u\left(S_{i}\right)$.

The probability of getting $A_{1}$ in this complex lottery is:

$$
P\left(A_{1}\right)=\sum_{i=1}^{n} P\left(A_{1} \mid S_{i}\right) \cdot P\left(S_{i}\right)=\sum_{i=1}^{n} u\left(S_{i}\right) \cdot p_{i} .
$$

In this complex lottery, we get:

- $A_{1}$ with probability $u=\sum_{i=1}^{n} p_{i} \cdot u\left(S_{i}\right)$, and

- $A_{0}$ with probability $1-u$.

So, the utility of the complex action is equal to the sum $u$.

From the mathematical viewpoint, the sum defining $u$ coincides with the expected value of the utility of an outcome. Thus, selecting the action with the largest utility means that we should select the action with the largest value of expected utility $u=\sum p_{i} \cdot u\left(S_{i}\right)$.

How uniquely determined is utility. The above definition of utility $u$ depends on the selection of two fixed alternatives $A_{0}$ and $A_{1}$. What if we use different alternatives $A_{0}^{\prime}$ and $A_{1}^{\prime}$ ? How will the new utility $u^{\prime}$ be related to the original utility $u$ ?

By definition of utility, every alternative $A$ is equivalent to a lottery $L(u(A))$ in which we get $A_{1}$ with probability $u(A)$ and $A_{0}$ with probability $1-u(A)$. For simplicity, let us assume that $A_{0}^{\prime}<A_{0}<A_{1}<A_{1}^{\prime}$. Then, for the utility $u^{\prime}$, we get $A_{0} \equiv L^{\prime}\left(u^{\prime}\left(A_{0}\right)\right)$ and $A_{1} \equiv L^{\prime}\left(u^{\prime}\left(A_{1}\right)\right)$. So, the alternative $A$ is equivalent to a complex lottery in which:

- we select $A_{1}$ with probability $u(A)$ and $A_{0}$ with probability $1-u(A)$; 
- depending on which of the two alternatives $A_{i}$ we get, we get $A_{1}^{\prime}$ with probability $u^{\prime}\left(A_{i}\right)$ and $A_{0}^{\prime}$ with probability $1-u^{\prime}\left(A_{i}\right)$.

In this complex lottery, we get $A_{1}^{\prime}$ with probability

$$
u^{\prime}(A)=u(A) \cdot\left(u^{\prime}\left(A_{1}\right)-u^{\prime}\left(A_{0}\right)\right)+u^{\prime}\left(A_{0}\right) .
$$

Thus, the utility $u^{\prime}(A)$ is related with the utility $u(A)$ by a linear transformation $u^{\prime}=$ $a \cdot u+b$, with $a>0$. In other words, utility is defined modulo a linear transformation.

Traditional approach summarized. We assume that

- we know possible actions, and

- we know the exact consequences of each action.

Then, we should select an action with the largest value of expected utility.

Empirical fact. It has been experimentally determined that for situations with monetary gain, utility $u$ grows with the money amount $x$ as $u \approx x^{\alpha}$, with $\alpha \approx 0.5$, i.e., approximately as $u \approx \sqrt{x}$; see, e.g., [8] and references therein.

What we do in this section. In this section, we explain this empirical dependence.

Main idea behind our explanation. Money is useful because one can buy goods and services with it. The more goods and services one buys, the better. In the first approximation, we can say that the utility increases with the increase in the number of goods and service.

In these terms, to estimate the utility corresponding to a given amount of money, we need to do two things:

- first, we need to estimate how many goods and services a person can buy for a given amount of money;

- second, we need to estimate what value of utility corresponds to this number of goods and services.

Step 1: estimating how many goods and services a person can buy. Different goods and services have different $\operatorname{costs} c_{i}$; some are cheaper, some are more expensive. We know that all the costs $c_{i}$ are bounded by some reasonable number $C$, so they are all located within an interval $[0, C]$. Let us sort the costs of different items in increasing order: $c_{1}<c_{2}<\ldots<c_{n}$.

In these terms, the smallest amount of money that we need to buy a single item is $c_{1}$. The smallest amount of money that we need to buy two items is $c_{1}+c_{2}$, etc. In general, the smallest amount of money that we need to buy $k$ items is $c_{1}+c_{2}+$ $\ldots+c_{k}$.

How does this amount depends on $k$ ? We do not know the exact costs $c_{i}$, all we know is that these costs are sorted in increasing order. Similarly to the previous section, we can therefore consider all possible combinations $c_{1}<\ldots<c_{n}$, and take, as an estimate $C_{i}$ for $c_{i}$, the average value of $c_{i}$ over all such combinations. Similarly to the previous section, we can conclude that $C_{i}=C \cdot \frac{i}{n+1}$. 
In these terms, the expected amount of money needed to buy $k$ items is equal to

$$
C_{1}+C_{2}+\ldots+C_{k}=\frac{C}{n} \cdot(1+2+\ldots+k)=\frac{C}{2 n} \cdot k \cdot(k+1) \approx \text { const } \cdot k^{2} .
$$

Step 2: estimating the utility corresponding to $k$ items. Let $u_{k}$ denote the utility corresponding to $k$ items. We know that all the values $u_{k}$ are bounded by some reasonable number $U$, so they are all located within an interval $[0, U]$. Clearly the more items, the better, i.e., the larger utility. Thus, we conclude that $u_{1}<u_{2}<\ldots<$ $u_{n}$.

We do not know the exact values of $u_{k}$, all we know is that these utility values are sorted in increasing order. We can thus consider all possible combinations $u_{1}<\ldots<u_{n}$, and take, as an estimate $U_{k}$ for $u_{k}$, the average value of $u_{k}$ over all such combinations. Similarly to the previous section, we can conclude that $U_{k}=U \cdot \frac{k}{n+1}=$ const $\cdot k$.

Let us combine these two estimates. What is the utility corresponding to the amount of money $x$ ? To answer this question, first, we estimate the number of items $k$ that we can buy with this amount. According to our estimates, $x=$ const $\cdot k^{2}$, so we conclude that $k=$ const $\cdot \sqrt{x}$. Then, we use this value $k$ to estimate the utility $U \approx U_{k}$. Substituting $k=$ const $\cdot \sqrt{x}$ into the formula $U \approx U_{k}=$ const $\cdot k$, we conclude that $U \approx$ const $\cdot \sqrt{x}$.

Since, as we have mentioned, utility is defined modulo a linear transformation, we can thus conclude that $U \approx \sqrt{x}$, which is exactly what we wanted to explain.

Conclusion. Thus, granularity indeed explains an interesting difficult-to-explain empirical fact - that utility grows as square root of money amount.

\section{Granularity Helps Reconcile Traditional Decision Making with Fuzzy Techniques}

Fuzzy uncertainty and fuzzy-based decision making. In addition to applying traditional decision theory, another very successful way of making decisions under uncertainty is to use techniques based on fuzzy logic and fuzzy uncertainty.

Fuzzy logic (see, e.g., [10, 21, 30]) has been designed to describe imprecise ("fuzzy") natural language properties like "big", "small", etc. In contrast to "crisp" properties like $x \leq 10$ which are either true or false, experts are not $100 \%$ sure whether a given value $x$ is big or small. To describe such properties $P$, fuzzy logic proposes to assign, to each possible value $x$, a degree $\mu_{P}(x)$ to which the value $x$ satisfies this property:

- the degree $\mu_{P}(x)=1$ means that we are absolutely sure that the value $x$ satisfies the property $P$; 
- the degree $\mu_{P}(x)=0$ means that we are absolutely sure that the value $x$ does not satisfy the property $P$; and

- intermediate degrees $0<\mu_{P}(x)<1$ mean that we have some confidence that $x$ satisfies the property $P$ but we also have a certain degree of confidence that the value $x$ does not satisfy this property.

How do we elicit the degree $\mu_{P}(x)$ from the expert? One of the usual ways is to use granules, i.e., more specifically, a Likert scale, i.e., to ask the expert to mark his or her degree of confidence that the value $x$ satisfies the property $P$ by one of the labels $0,1, \ldots, n$ on a scale from 0 to $n$. If an expert marks $m$ on a scale from 0 to $n$, then we take the ratio $m / n$ as the desired degree $\mu_{P}(x)$. For example, if an expert marks her confidence by a value 7 on a scale from 0 to 10 , then we take $\mu_{P}(x)=7 / 10$.

For a fixed scale from 0 to $n$, we only get $n+1$ values this way: $0,1 / n, 2 / n, \ldots$, $(n-1) / n=1-1 / n$, and 1 . If we want a more detailed description of the expert's uncertainty, we can use a more detailed scale, with a larger value $n$.

Problem: how to reconcile traditional decision making theory with fuzzy techniques? The traditional decision theory describes rational human decision making, it has many practical applications. On the other hand, fuzzy techniques are also very successful in many application problems, in particular, in control and in decision making (see, e.g., [10, 21]).

It is therefore desirable to combine these two techniques, so that we would able to capitalize on the successes of both types of techniques. To enhance this combination, it is desirable to be able to describe both techniques in the same terms. In particular, it is desirable to describe fuzzy uncertainty in terms of traditional decision making.

How do we select a mark on a Likert scale? In Section 3, we simply used the labels marked by people on a Likert scale. But how do people select which labels to mark? To understand this, let us recall how this marking is done. Suppose that we have a Likert scale with $n+1$ labels $0,1,2, \ldots, n$, ranging from the smallest to the largest.

Then, if the actual value of the quantity $x$ is very small, we mark label 0 . At some point, we change to label 1 ; let us mark this threshold point by $x_{1}$. When we continue increasing $x$, we first have values marked by label 1 , but eventually reach a new threshold after which values will be marked by label 2; let us denote this threshold by $x_{2}$, etc. As a result, we divide the range $[\underline{X}, \bar{X}]$ of the original variable into $n+1$ intervals $\left[x_{0}, x_{1}\right],\left[x_{1}, x_{2}\right], \ldots,\left[x_{n-1}, x_{n}\right],\left[x_{n}, x_{n+1}\right]$, where $x_{0}=\underline{X}$ and $x_{n+1}=\bar{X}$ :

- values from the first interval $\left[x_{0}, x_{1}\right]$ are marked with label 0 ;

- values from the second interval $\left[x_{1}, x_{2}\right]$ are marked with label 1 ;

- ...

- values from the $n$-th interval $\left[x_{n-1}, x_{n}\right]$ are marked with label $n-1$;

- values from the $(n+1)$-st interval $\left[x_{n}, x_{n+1}\right]$ are marked with label $n$.

Then, when we need to make a decision, we base this decision only on the label, i.e., only on the interval to which $x$ belongs. In other words, we make $n$ different 
decisions depending on whether $x$ belongs to the interval $\left[x_{0}, x_{1}\right]$, to the interval $\left[x_{1}, x_{2}\right], \ldots$, or to the interval $\left[x_{n}, x_{n+1}\right]$.

Decisions based on the Likert discretization are imperfect. Ideally, we should take into account the exact value of the variable $x$. When we use Likert scale, we only take into account an interval containing $x$ and thus, we do not take into account part of the original information. Since we only use part of the original information about $x$, the resulting decision may not be as good as the decision based on the ideal complete knowledge.

For example, an ideal office air conditioner should be able to maintain the exact temperature at which a person feels comfortable. People are different, their temperature preferences are different, so an ideal air conditioner should be able to maintain any temperature value $x$ within a certain range $[\underline{X}, \bar{X}]$. In practice, some air conditioners only have a finite number of settings. For example, if we have setting corresponding to $65,70,75$, and 80 degrees, then a person who prefers 72 degrees will probably select the 70 setting or the 75 setting. In both cases, this person will be somewhat less comfortable than if there was a possibility of an ideal 72 degrees setting.

How do we select a Likert scale: main idea. According to the general ideas of traditional (utility-based) approach to decision making, we should select a Likert scale for which the expected utility is the largest.

To estimate the utility of decisions based on each scale, we will take into account the just-mentioned fact that decisions based on the Likert discretization are imperfect. In utility terms, this means that the utility of the Likert-based decisions is, in general, smaller than the utility of the ideal decision.

Which decision should we choose within each label? In the ideal situation, if we could use the exact value of the quantity $x$, then for each value $x$, we would select an optimal decision $d(x)$, a decision which maximizes the person's utility.

If we only know the label $k$, i.e., if we only know that the actual value $x$ belongs to the $(k+1)$-st interval $\left[x_{k}, x_{k+1}\right]$, then we have to make a decision based only on this information. In other words, we have to select one of the possible values $\widetilde{x}_{k} \in\left[x_{k}, x_{k+1}\right]$, and then, for all $x$ from this interval, use the decision $d\left(\widetilde{x}_{k}\right)$ based on this value.

Which value $\widetilde{x}_{k}$ should we choose: idea. According to the traditional approach to decision making, we should select a value for which the expected utility is the largest.

Which value $\widetilde{x}_{k}$ should we choose: towards a precise formulation of the problem. To find this expected utility, we need to know two things:

- we need to know the probability of different values of $x$; these probabilities can be described, e.g., by the probability density function $\rho(x)$;

- we also need to know, for each pair of values $x^{\prime}$ and $x$, what is the utility $u\left(x^{\prime}, x\right)$ of using a decision $d\left(x^{\prime}\right)$ in the situation in which the actual value is $x$. 
In these terms, the expected utility of selecting a value $\widetilde{x}_{k}$ can be described as

$$
\int_{x_{k}}^{x_{k+1}} \rho(x) \cdot u\left(\widetilde{x}_{k}, x\right) d x
$$

Thus, for each interval $\left[x_{k}, x_{k+1}\right]$, we need to select a decision $d\left(\widetilde{x}_{k}\right)$ corresponding to the value $\tilde{x}_{k}$ for which the expression (5.1) attains its largest possible value. The resulting expected utility is equal to

$$
\max _{\tilde{x}_{k}} \int_{x_{k}}^{x_{k+1}} \rho(x) \cdot u\left(\widetilde{x}_{k}, x\right) d x .
$$

How to select the best Likert scale: general formulation of the problem. The actual value $x$ can belong to any of the $n+1$ intervals $\left[x_{k}, x_{k+1}\right]$. Thus, to find the overall expected utility, we need to add the values (5.2) corresponding to all these intervals. In other words, we need to select the values $x_{1}, \ldots, x_{n}$ for which the following expression attains its largest possible value:

$$
\sum_{k=0}^{n} \max _{\tilde{x}_{k}} \int_{x_{k}}^{x_{k+1}} \rho(x) \cdot u\left(\widetilde{x}_{k}, x\right) d x .
$$

Equivalent reformulation in terms of disutility. In the ideal case, for each value $x$, we should use a decision $d(x)$ corresponding to this value $x$, and gain utility $u(x, x)$. In practice, we have to use decisions $d\left(x^{\prime}\right)$ corresponding to a slightly different value, and thus, get slightly worse utility values $u\left(x^{\prime}, x\right)$. The corresponding decrease in utility $U\left(x^{\prime}, x\right) \stackrel{\text { def }}{=} u(x, x)-u\left(x^{\prime}, x\right)$ is usually called disutility. In terms of disutility, the function $u\left(x^{\prime}, x\right)$ has the form

$$
u\left(x^{\prime}, x\right)=u(x, x)-U\left(x^{\prime}, x\right)
$$

and thus, the optimized expression (5.1) takes the form

$$
\int_{x_{k}}^{x_{k+1}} \rho(x) \cdot u(x, x) d x-\int_{x_{k}}^{x_{k+1}} \rho(x) \cdot U\left(\widetilde{x}_{k}, x\right) d x .
$$

The first integral does not depend on $\widetilde{x}_{k}$; thus, the expression (5.1) attains its maximum if and only if the second integral attains its minimum. The resulting maximum (5.2) thus takes the form

$$
\int_{x_{k}}^{x_{k+1}} \rho(x) \cdot u(x, x) d x-\min _{\tilde{x}_{k}} \int_{x_{k}}^{x_{k+1}} \rho(x) \cdot U\left(\widetilde{x}_{k}, x\right) d x .
$$

Thus, the expression (5.3) takes the form

$$
\sum_{k=0}^{n} \int_{x_{k}}^{x_{k+1}} \rho(x) \cdot u(x, x) d x-\sum_{k=0}^{n} \min _{\tilde{x}_{k}} \int_{x_{k}}^{x_{k+1}} \rho(x) \cdot U\left(\widetilde{x}_{k}, x\right) d x .
$$


The first sum does not depend on selecting the thresholds. Thus, to maximize utility, we should select the values $x_{1}, \ldots, x_{n}$ for which the second sum attains its smallest possible value:

$$
\sum_{k=0}^{n} \min _{\tilde{x}_{k}} \int_{x_{k}}^{x_{k+1}} \rho(x) \cdot U\left(\widetilde{x}_{k}, x\right) d x \rightarrow \min
$$

Let is recall that are interested in the membership function. For a general Likert scale, we have a complex optimization problem (5.5). However, we are not interested in general Likert scales per se, what we are interested in is the use of Likert scales to elicit the values of the membership function $\mu(x)$.

As we have mentioned earlier, in an $n$-valued scale:

- the smallest label 0 corresponds to the value $\mu(x)=0 / n$,

- the next label 1 corresponds to the value $\mu(x)=1 / n$,

- ...

- the last label $n$ corresponds to the value $\mu(x)=n / n=1$.

Thus, for each $n$ :

- values from the interval $\left[x_{0}, x_{1}\right]$ correspond to the value $\mu(x)=0 / n$;

- values from the interval $\left[x_{1}, x_{2}\right]$ correspond to the value $\mu(x)=1 / n$;

- ...

- values from the interval $\left[x_{n}, x_{n+1}\right]$ correspond to the value $\mu(x)=n / n=1$.

The actual value of the membership function $\mu(x)$ corresponds to the limit $n \rightarrow \infty$, i.e., in effect, to very large values of $n$. Thus, in our analysis, we will assume that the number $n$ of labels is huge - and thus, that the width of each of $n+1$ intervals $\left[x_{k}, x_{k+1}\right]$ is very small.

Let us take into account that each interval is narrow. Let us use the fact that each interval is narrow to simplify the expression $U\left(x^{\prime}, x\right)$ and thus, the optimized expression (5.5).

In the expression $U\left(x^{\prime}, x\right)$, both values $x^{\prime}$ and $x$ belong to the same narrow interval and thus, the difference $\Delta x \stackrel{\text { def }}{=} x^{\prime}-x$ is small. Thus, we can expand the expression $U\left(x^{\prime}, x\right)=U(x+\Delta x, x)$ into Taylor series in $\Delta x$, and keep only the first non-zero term in this expansion. In general, we have

$$
U(x+\Delta, x)=U_{0}(x)+U_{1} \cdot \Delta x+U_{2}(x) \cdot \Delta x^{2}+\ldots,
$$

where

$$
U_{0}(x)=U(x, x), U_{1}(x)=\frac{\partial U(x+\Delta x, x)}{\partial(\Delta x)}, U_{2}(x)=\frac{1}{2} \cdot \frac{\partial^{2} U(x+\Delta x, x)}{\partial^{2}(\Delta x)} .
$$

Here, by definition of disutility, we get $U_{0}(x)=U(x, x)=u(x, x)-u(x, x)=0$. Since the utility is the largest (and thus, disutility is the smallest) when $x^{\prime}=x$, i.e., when $\Delta x=0$, the derivative $U_{1}(x)$ is also equal to 0 - since the derivative of each (differentiable) function is equal to 0 when this function attains its minimum. Thus, the 
first non-trivial term corresponds to the second derivative:

$$
U(x+\Delta x, x) \approx U_{2}(x) \cdot \Delta x^{2},
$$

i.e., in other words, that

$$
U\left(\widetilde{x}_{k}, x\right) \approx U_{2}(x) \cdot\left(\widetilde{x}_{k}-x\right)^{2} .
$$

Substituting this expression into the expression

$$
\int_{x_{k}}^{x_{k+1}} \rho(x) \cdot U\left(\widetilde{x}_{k}, x\right) d x
$$

that needs to be minimized if we want to find the optimal $\widetilde{x}_{k}$, we conclude that we need to minimize the integral

$$
\int_{x_{k}}^{x_{k+1}} \rho(x) \cdot U_{2}(x) \cdot\left(\widetilde{x}_{k}-x\right)^{2} d x .
$$

This new integral is easy to minimize: if we differentiate this expression with respect to the unknown $\widetilde{x}_{k}$ and equate the derivative to 0 , we conclude that

$$
\int_{x_{k}}^{x_{k+1}} \rho(x) \cdot U_{2}(x) \cdot\left(\widetilde{x}_{k}-x\right) d x=0,
$$

i.e., that

$$
\tilde{x}_{k} \cdot \int_{x_{k}}^{x_{k+1}} \rho(x) \cdot U_{2}(x) d x=\int_{x_{k}}^{x_{k+1}} x \cdot \rho(x) \cdot U_{2}(x) d x,
$$

and thus, that

$$
\widetilde{x}_{k}=\frac{\int_{x_{k}}^{x_{k+1}} x \cdot \rho(x) \cdot U_{2}(x) d x}{\int_{x_{k}}^{x_{k+1}} \rho(x) \cdot U_{2}(x) d x} .
$$

This expression can also be simplified if we take into account that the intervals are narrow. Specifically, if we denote the midpoint of the interval $\left[x_{k}, x_{k+1}\right]$ by $\bar{x}_{k} \stackrel{\text { def }}{=} \frac{x_{k}+x_{k+1}}{2}$, and denote $\Delta x \stackrel{\text { def }}{=} x-\bar{x}_{k}$, then we have $x=\bar{x}_{k}+\Delta x$. Expanding the corresponding expressions into Taylor series in terms of a small value $\Delta x$ and keeping only main terms in this expansion, we get

$$
\rho(x)=\rho\left(\bar{x}_{k}+\Delta x\right)=\rho\left(\bar{x}_{k}\right)+\rho^{\prime}\left(\bar{x}_{k}\right) \cdot \Delta x \approx \rho\left(\bar{x}_{k}\right),
$$

where $f^{\prime}(x)$ denoted the derivative of a function $f(x)$, and

$$
U_{2}(x)=U_{2}\left(\bar{x}_{k}+\Delta x\right)=U_{2}\left(\bar{x}_{k}\right)+U_{2}^{\prime}\left(\bar{x}_{k}\right) \cdot \Delta x \approx U_{2}\left(\bar{x}_{k}\right) .
$$

Substituting these expressions into the formula (5.9), we conclude that 


$$
\tilde{x}_{k}=\frac{\rho\left(\bar{x}_{k}\right) \cdot U_{2}\left(\bar{x}_{k}\right) \cdot \int_{x_{k}}^{x_{k+1}} x d x}{\rho\left(\bar{x}_{k}\right) \cdot U_{2}\left(\bar{x}_{k}\right) \cdot \int_{x_{k}}^{x_{k+1}} d x}=\frac{\int_{x_{k}}^{x_{k+1}} x d x}{\int_{x_{k}}^{x_{k+1}} d x}=\frac{\frac{1}{2} \cdot\left(x_{k+1}^{2}-x_{k}^{2}\right)}{x_{k+1}-x_{k}}=\frac{x_{k+1}+x_{k}}{2}=\bar{x}_{k} .
$$

Substituting this midpoint value $\widetilde{x}_{k}=\bar{x}_{k}$ into the integral (5.8) and taking into account that on the $k$-th interval, we have $\rho(x) \approx \rho\left(\bar{x}_{k}\right)$ and $U_{2}(x) \approx U_{2}\left(\bar{x}_{k}\right)$, we conclude that the integral (5.8) takes the form

$$
\int_{x_{k}}^{x_{k+1}} \rho\left(\bar{x}_{k}\right) \cdot U_{2}\left(\bar{x}_{k}\right) \cdot\left(\bar{x}_{k}-x\right)^{2} d x=\rho\left(\bar{x}_{k}\right) \cdot U_{2}\left(\bar{x}_{k}\right) \cdot \int_{x_{k}}^{x_{k+1}}\left(\bar{x}_{k}-x\right)^{2} d x .
$$

When $x$ goes from $x_{k}$ to $x_{k+1}$, the difference $\Delta x=x-\bar{x}_{k}$ between the value $x$ and the interval's midpoint $\bar{x}_{k}$ ranges from $-\Delta_{k}$ to $\Delta_{k}$, where $\Delta_{k}$ is the interval's half-width:

$$
\Delta_{k} \stackrel{\text { def }}{=} \frac{x_{k+1}-x_{k}}{2} \text {. }
$$

In terms of the new variable $\Delta x$, the integral in the right-hand side of (5.8a) has the form

$$
\int_{x_{k}}^{x_{k+1}}\left(\bar{x}_{k}-x\right)^{2} d x=\int_{-\Delta_{k}}^{\Delta_{k}}(\Delta x)^{2} d(\Delta x)=\frac{2}{3} \cdot \Delta_{k}^{3} .
$$

Thus, the integral (5.8) takes the form

$$
\frac{2}{3} \cdot \rho\left(\bar{x}_{k}\right) \cdot U_{2}\left(\bar{x}_{k}\right) \cdot \Delta_{k}^{3}
$$

The problem (5.5) of selecting the Likert scale thus becomes the problem of minimizing the sum (5.5) of such expressions (5.8), i.e., of the sum

$$
\frac{2}{3} \cdot \sum_{k=0}^{n} \rho\left(\bar{x}_{k}\right) \cdot U_{2}\left(\bar{x}_{k}\right) \cdot \Delta_{k}^{3}
$$

Here, $\bar{x}_{k+1}=x_{k+1}+\Delta_{k+1}=\left(\bar{x}_{k}+\Delta_{k}\right)+\Delta_{k+1} \approx \bar{x}_{k}+2 \Delta_{k}$, so $\Delta_{k}=(1 / 2) \cdot \Delta \bar{x}_{k}$, where $\Delta \bar{x}_{k} \stackrel{\text { def }}{=} \bar{x}_{k+1}-\bar{x}_{k}$. Thus, (5.10) takes the form

$$
\frac{1}{3} \cdot \sum_{k=0}^{n} \rho\left(\bar{x}_{k}\right) \cdot U_{2}\left(\bar{x}_{k}\right) \cdot \Delta_{k}^{2} \cdot \Delta \bar{x}_{k}
$$

In terms of the membership function, we have $\mu\left(\bar{x}_{k}\right)=k / n$ and $\mu\left(\bar{x}_{k+1}\right)=(k+$ 1) $/ n$. Since the half-width $\Delta_{k}$ is small, we have

$$
\frac{1}{n}=\mu\left(\bar{x}_{k+1}\right)-\mu\left(\bar{x}_{k}\right)=\mu\left(\bar{x}_{k}+2 \Delta_{k}\right)-\mu\left(\bar{x}_{k}\right) \approx \mu^{\prime}\left(\bar{x}_{k}\right) \cdot 2 \Delta_{k},
$$

thus, $\Delta_{k} \approx \frac{1}{2 n} \cdot \frac{1}{\mu^{\prime}\left(\bar{x}_{k}\right)}$. Substituting this expression into (5.11), we get the expression $\frac{1}{3 \cdot(2 n)^{2}} \cdot I$, where 


$$
I=\sum_{k=0}^{n} \frac{\rho\left(\bar{x}_{k}\right) \cdot U_{2}\left(\bar{x}_{k}\right)}{\left(\mu^{\prime}\left(\bar{x}_{k}\right)\right)^{2}} \cdot \Delta \bar{x}_{k} .
$$

The expression $I$ is an integral sum, so when $n \rightarrow \infty$, this expression tends to the corresponding integral

$$
I=\int \frac{\rho(x) \cdot U_{2}(x)}{\left(\mu^{\prime}(x)\right)^{2}} d x .
$$

Minimizing (5.5) is equivalent to minimizing $I$. With respect to the derivative $d(x) \stackrel{\text { def }}{=} \mu^{\prime}(x)$, we need to minimize the objective function

$$
I=\int \frac{\rho(x) \cdot U_{2}(x)}{d^{2}(x)} d x
$$

under the constraint that

$$
\int_{\underline{X}}^{\bar{X}} d(x) d x=\mu(\bar{X})-\mu(\underline{X})=1-0=1 .
$$

By using the Lagrange multiplier method, we can reduce this constraint optimization problem to the unconstrained problem of minimizing the functional

$$
I=\int \frac{\rho(x) \cdot U_{2}(x)}{d^{2}(x)} d x+\lambda \cdot \int d(x) d x,
$$

for an appropriate Lagrange multiplier $\lambda$. Differentiating (5.14) with respect to $d(x)$ and equating the derivative to 0 , we conclude that $-2 \cdot \frac{\rho(x) \cdot U_{2}(x)}{d^{3}(x)}+\lambda=0$, i.e., that $d(x)=c \cdot\left(\rho(x) \cdot U_{2}(x)\right)^{1 / 3}$ for some constant $c$. Thus, $\mu(x)=\int_{X}^{x} d(t) d t=c \cdot \int_{X}^{x}(\rho(t)$. $\left.U_{2}(t)\right)^{1 / 3} d t$. The constant $c$ must be determined by the condition that $\mu(\bar{X})=1$. Thus, we arrive at the following formula (5.15).

Resulting formula. The membership function $\mu(x)$ obtained by using Likert-scale elicitation is equal to

$$
\mu(x)=\frac{\int_{\underline{X}}^{x}\left(\rho(t) \cdot U_{2}(t)\right)^{1 / 3} d t}{\int_{\underline{X}}^{\bar{X}}\left(\rho(t) \cdot U_{2}(t)\right)^{1 / 3} d t},
$$

where $\rho(x)$ is the probability density describing the probabilities of different values of $x$,

$$
U_{2}(x) \stackrel{\text { def }}{=} \frac{1}{2} \cdot \frac{\partial^{2} U(x+\Delta x, x)}{\partial^{2}(\Delta x)}
$$

$U\left(x^{\prime}, x\right) \stackrel{\text { def }}{=} u(x, x)-u\left(x^{\prime}, x\right)$, and $u\left(x^{\prime}, x\right)$ is the utility of using a decision $d\left(x^{\prime}\right)$ corresponding to the value $x^{\prime}$ in the situation in which the actual value is $x$.

Comment. The above formula only applies to membership functions like "large" whose values monotonically increase with $x$. It is easy to write a similar formula for membership functions like "small" which decrease with $x$. For membership func- 
tions like "approximately 0" which first increase and then decrease, we need to separately apply these formula to both increasing and decreasing parts.

Conclusion. The resulting membership degrees incorporate both probability and utility information. This fact explains why fuzzy techniques often work better than probabilistic techniques - because the probability techniques only take into account the probability of different outcomes.

\section{Conclusions and Future Work}

While in general, humans behave rationally, there are many known experiments in which humans show seemingly irrational behavior. For example, when a customer is presented with two objects, one somewhat cheaper and another one more expensive and of higher quality, the customer's choice often depend on the presence of the third object, the object that the customer will not select:

- if the third object is cheaper than both two, the customer will usually select the cheaper of the two objects;

- if the third object is more expensive than the both two, the customer will usually select the more expensive of the two objects.

From the rational viewpoint, the selection between the two object should not depend on the presence of other, less favorable objects - but it does!

There are many other examples of such seemingly irrational human behavior. This phenomenon is known since the 1950s, and an explanation for this phenomenon is also well known - such seemingly irrational behavior is caused by the fact that human computational abilities are limited; in this sense, human rationality is bounded.

The idea of bounded rationality explains, on the qualitative level, why human behavior and decision making are sometimes seemingly irrational. However, until recently, there have been few successful attempts to use this idea to explain quantitative aspects of observed human behavior. In this chapter, we show, on four examples, that these quantitative aspects can be explained if we take into account that one of the main consequences of bounded rationality is granularity. The main idea behind granularity is that since we cannot process all the available information, we only process part of it. Because of this, several different data points - differing by the information that we do not process - are treated the same way. In other words, instead of dealing with the original data points, we deal with granules, each of which corresponds to several possible data points. For example, if we only use the first binary digit $x_{1}$ in the binary expansion of a number $x=0 . x_{1} x_{2} \ldots$ from the interval $[0,1]$, this means that, instead of the exact number $x$, we use two granules corresponding to intervals $[0,0.5)$ (for which $x_{1}=0$ ) and $[0.5,1]$ (for which $x_{1}=1$ ).

In this chapter, we have shown, on four examples (including the above customer examples) that granularity indeed explained the observed quantitative aspects of 
seemingly irrational human behavior. The remaining challenge is to provide a similar explanation for other observed cases of seemingly irrational human behavior and decision making.

Acknowledgements This work was supported in part by the National Science Foundation grants HRD-0734825 and HRD-1242122 (Cyber-ShARE Center of Excellence) and DUE-0926721.

The authors are thankful to participants of the Joint World Congress of the International Fuzzy Systems Association and Annual Conference of the North American Fuzzy Information Processing Society IFSA/NAFIPS'2013 (Edmonton, Canada, June 24-28, 2013), 4th World Conference on Soft Computing (Berkeley, California, May 25-27, 2014), and 2014 Annual Conference of the North American Fuzzy Information Processing Society NAFIPS'2014 (Boston, Massachusetts, June 24-26, 2014) for valuable discussions, and to the anonymous referees for useful suggestions.

\section{References}

1. Ahsanullah, M., Nevzorov, V.B., Shakil, M.: An Introduction to Order Statistics, Atlantis Press, Paris (2013)

2. Arnold, B.C., Balakrishnan, N., Nagaraja, H.N.: A First Course in Order Statistics, Society of Industrial and Applied Mathematics (SIAM), Philadelphia, Pennsylvania (2008)

3. Bellman, R.E., Zadeh, L.A.: Decision making in a fuzzy environment. Management Science 17(4), B141-B164 (1970)

4. Cohen, M.D., Huber, G., Keeney, R.L., Levis, A.H., Lopes, L.L., Sage, A.P., Sen, S., Whinston, A.B., Winkler, R.L., von Winterfeldt, D., Zadeh, L.A.: Research needs and the phenomena of decision making and operations. IEEE Transactions on Systems, Man, and Cybernetics 15(6), 764-775 (1985)

5. David, H.A., Nagaraja, H.N.: Order Statistics, Wiley, New York (2003)

6. Fishburn, P.C.: Utility Theory for Decision Making, John Wiley \& Sons Inc., New York (1969)

7. Fishburn, P.C.: Nonlinear Preference and Utility Theory, The John Hopkins Press, Baltimore, Maryland (1988)

8. Kahneman, D.: Thinking, Fast and Slow, Farrar, Straus, and Giroux, New York (2011)

9. Keeney, R.L., Raiffa, H.: Decisions with Multiple Objectives, John Wiley and Sons, New York (1976)

10. Klir, G., Yuan, B.: Fuzzy Sets and Fuzzy Logic, Prentice Hall, Upper Saddle River, New Jersey (1995)

11. Kreinovich, V.: Decision making under interval uncertainty (and beyond), In: Guo, P., Pedrycz W. (eds.): Human-Centric Decision-Making Models for Social Sciences, Springer Verlag, pp. 163-193 (2014)

12. Lorkowski, J., Kreinovich, V.: Likert-scale fuzzy uncertainty from a traditional decision making viewpoint: it incorporates both subjective probabilities and utility information. In: Proceedings of the Joint World Congress of the International Fuzzy Systems Association and Annual Conference of the North American Fuzzy Information Processing Society IFSA/NAFIPS'2013, Edmonton, Canada, June 24-28, 2013, pp. 525-530 (2013)

13. Lorkowski, J., Kreinovich, V.: Fuzzy logic ideas can help in explaining Kahneman and Tversky's empirical decision weights. In: Proceedings of the 4th World Conference on Soft Computing, Berkeley, California, May 25-27, 2014, pp. 285-289 (2014)

14. Lorkowski, J., Kreinovich, V.: Interval and symmetry approaches to uncertainty - pioneered by Wiener - help explain seemingly irrational human behavior: a case study, In: Proceedings of the 2014 Annual Conference of the North American Fuzzy Information Processing Society NAFIPS'2014, Boston, Massachusetts, June 24-26, 2014 (2014)

15. Luce, R.D., Raiffa, R.: Games and Decisions: Introduction and Critical Survey, Dover, New York (1989) 
16. March, J.: Bounded rationality, ambiguity, and the engineering of choice. The Bell Journal of Economics 9(2), 587-608 (1978)

17. Nguyen, H.T., Kosheleva, O., Kreinovich, V.: Decision making beyond Arrow's "Impossibility Theorem", with the analysis of effects of collusion and mutual attraction. International Journal of Intelligent Systems 24(1), 27-45 (2009)

18. Nguyen, H.T., Kreinovich, V.: Applications of Continuous Mathematics to Computer Science, Kluwer, Dordrecht (1997)

19. Nguyen, H.T., Kreinovich, V., Lea, B.: How to combine probabilistic and fuzzy uncertainties in fuzzy control. In: Proceedings of the Second International Workshop on Industrial Applications of Fuzzy Control and Intelligent Systems, College Station, December 2-4, 1992, pp. 117-121 (1992)

20. Nguyen, H.T., Kreinovich, V., Wu, B., Xiang, G.: Computing Statistics under Interval and Fuzzy Uncertainty, Springer Verlag, Berlin, Heidelberg (2012)

21. Nguyen, H.T., Walker, E.A.: A First Course in Fuzzy Logic, Chapman and Hall/CRC, Boca Raton, Florida (2006)

22. Pedrycz, W., Skowron, A., Kreinovich, V. (eds.): Handbook on Granular Computing, Wiley, Chichester, UK (2008)

23. Rabinovich, S.G.: Measurement Errors and Uncertainty: Theory and Practice, Springer Verlag, Berlin (2005)

24. Raiffa, H.: Decision Analysis, McGraw-Hill, Columbus, Ohio (1997)

25. Redelmeier, D., Shafir, E.: Medical decision mading in situations that offer multiple alternatives. Journal of the American Medical Association 273(4), 302-305 (1995)

26. Shafir, E., Simonson, I., Tversky, A.: Reason-based choice. Cognition 49, 11-36 (1993)

27. Sheskin, D.J.: Handbook of Parametric and Nonparametric Statistical Procedures, Chapman \& Hall/CRC, Boca Raton, Florida, (2011)

28. Simonson, I., Rosen, E.: Absolute Value: What Really Influences Customers in the Age of (Nearly) Perfect Information, HarperBusiness, New York (2014)

29. Tversky, A., Simonson, I.: Context-dependent preferences. Management Science 39(10), 1179-1189 (1993)

30. Zadeh, L.A.: Fuzzy sets. Information and Control 8, 338-353 (1965)

31. Zadeh, L.A.: Outline of a new approach to the analysis of complex systems and decision processes. IEEE Transactions on Systems, Man, and Cybernetics 3(1), 28-44 (1973)

32. Zadeh, L.A.: Precisiated natural language-toward a radical enlargement of the role of natural languages in information processing, decision and control, In: Wang, L., Halgamuge, S.K., Yao, X. (eds.): Proceedings of the 1st International Conference on Fuzzy Systems and Knowledge Discovery FSDK'02: Computational Intelligence for the E-Age, Singapore, November 18-22, 2002, vol. 1, pp. 1-3 (2002)

33. Zadeh, L.A.: Computing with words and perceptions - a paradigm shift in computing and decision analysis and machine intelligence, In: Wani, A., Cios, K.J., Hafeez K. (eds.): Proceedings of the 2003 International Conference on Machine Learning and Applications ICMLA'2003, Los Angeles, California, June 23-24, 2003, pp. 3-5 (2003)

34. Zadeh, L.A.: A new direction in decision analysis-perception-based decisions”, In: Ralescu, A.L. (ed.): Proceedings of the Fourteenth Midwest Artificial Intelligence and Cognitive Sciences Conference MAICS'2003, Cincinnati, Ohio, April 12-13, 2003, pp. 1-2 (2003) 